\title{
Innovation in user-centered skills and performance improvement for sustainable complex service systems
}

\author{
Waldemar Karwowski a,b , Tareq Z. Ahram ${ }^{\mathrm{a}, \mathrm{b}^{*} \text {, }}$ \\ ${ }^{a}$ Institute for Advanced Systems Engineering, , Orlando, FL 32816, USA \\ ${ }^{b}$ Department of Industrial Engineering and Management Systems, University of Central Florida, Orlando, FL \\ 32816, USA
}

\begin{abstract}
In order to leverage individual and organizational learning and to remain competitive in current turbulent markets it is important for employees, managers, planners and leaders to perform at high levels over time. Employee competence and skills are extremely important matters in view of the general shortage of talent and the mobility of employees with talent. Two factors emerged to have the greatest impact on the competitiveness of complex service systems: improving managerial and employee's knowledge attainment for skills, and improving the training and development of the workforce. This paper introduces the knowledge-based user-centered service design approach for sustainable skill and performance improvement in education, design and modeling of the next generation of complex service systems. The rest of the paper cover topics in human factors and sustainable business process modeling for the service industry, and illustrates the user-centered service system development cycle with the integration of systems engineering concepts in service systems. A roadmap for designing service systems of the future is discussed. The framework introduced in this paper is based on key user-centered design principles and systems engineering applications to support service competitiveness.
\end{abstract}

Keywords: Service Systems, Performance, Sustainability, Ontology

\section{Introduction}

Businesses thrive to differentiate themselves under pressures of rapid technological change, competitors, and regulatory challenges. These pressures force companies to continuously search for new technologies that help improve their internal and external processes to reduce the time required to market new or improved products and services. The most prominent issue is the lack of automation when trying to integrate new business requirements into existing services and systems, adding new components and adapting existing processes as priorities and perspectives change. Increased complexity of service systems and the underlying knowledge required for those systems has influenced industrial and economic growth in many nations. These developments have reinforced the need for emphasizing the role of information and knowledge in service systems. Service systems sparked a revolution whereby the traditional industrial society that emerged over the last two cen- turies is being rapidly overtaken by the new information society $[10,2]$. The design of complex service systems considers qualitative attributes between human-human and human-machine interactions. These considerations encompass service employees and associates (i.e., those who provide the service) as well as service users or stakeholders (i.e., those who receive and use the service). The service systems design process also includes the necessary objects and/or components that constitute successful business decisions and therefore competitive service offering.

User-centered design (UCD) is a proactive approach for making informed and appropriate design decisions. Executives may feel pressured to make quick, unfounded, and aggressive business decisions. Such impulsive choices can be disastrous in detailed and far-reaching design activities. Business Process Modeling Ontology (BPMO) defines the link between processes and organizations in an effort to enhance the decision making activities. Business process modeling through UCD provides adequate

*Corresponding author. E-mail: tahram@ucf.edu 
data to make rational and substantiated decisions regarding business process management actions, user experience choices, and usability of services and products. A reactive approach to feedback is no longer adequate. Key decisions must ensure that the root causes of problems are addressed and that a usercentric methodology be employed. According to Nadel and Piazza [14], two of the many justifications for employing a user-centric method are:

1) Users of the service often have unspoken needs that are hard to articulate. Through systematic data gathering, a skilled analyst can uncover how users conceptually think about a task. Only then is it possible to design an intuitive navigational scheme that reflects this mental model.

2) Usability problems are often symptomatic of deeper business issues.

A major consumer electronics company found that its e-commerce site was drastically underperforming that of the competition's site. It turned out that the site was confusing customers due to its structure. The site was organized around the company's separate business units for internal political reasons. Each division was so territorial that they couldn't agree on a unified site structure-each wanted their own homepage. As a result, customers couldn't compare and order a product from the same section of the site. If they found a television matching their preference, they had to return to the main corporate homepage and then navigate to another area to actually buy it!

UCD requires stakeholder analysis to determine which business needs are being addressed and what the organization really wants to achieve. A design that attempts to achieve business objectives but ignores the actual users is bound to fail. The Center for Business Knowledge has identified four pillars of knowledge management, shown in Figure 1: People, Process, Culture and Technology. With process and user knowledge as a foundation, this will enable conducting end-user data gathering. The insight this provides about user behavior let us design an interface/architecture that is intuitive and logical. Success must ultimately be measured against the following parameters: does the site/application meet business needs and can users efficiently accomplish what they want to do?

Management of sustainable complex service systems are characterized into four main dimensions [6, 13]:
1) Structure: human, material, information, communication, technology, resources, and operating facilities

2) Process: process model, service provision

3) Outcome: product model, service content, consequences, quality, performance and standards

4) Market: requirement model, market requirements and User needs

Although the field of user-centered design is wellsuited for evaluation and analysis, many service systems designers stumble when using user-centered approaches as tools for iterating process design solutions. Human Factors Engineering (HFE) and Usability Evaluation (UE) often occur too late in the design process to effect meaningful change, or changes cannot be made due to cost or schedule. However, HFE and usability require some semblance of a design to analyze, so evaluations cannot be implemented from the very start either. To mediate this, heuristics general HFE design practices should be employed at conceptual stages, with appropriate HFE and UE practices occurring at service maturity levels. Such early intervention and ongoing iterative evaluation would greatly aid in the design and execution of existing business process service prototypes.

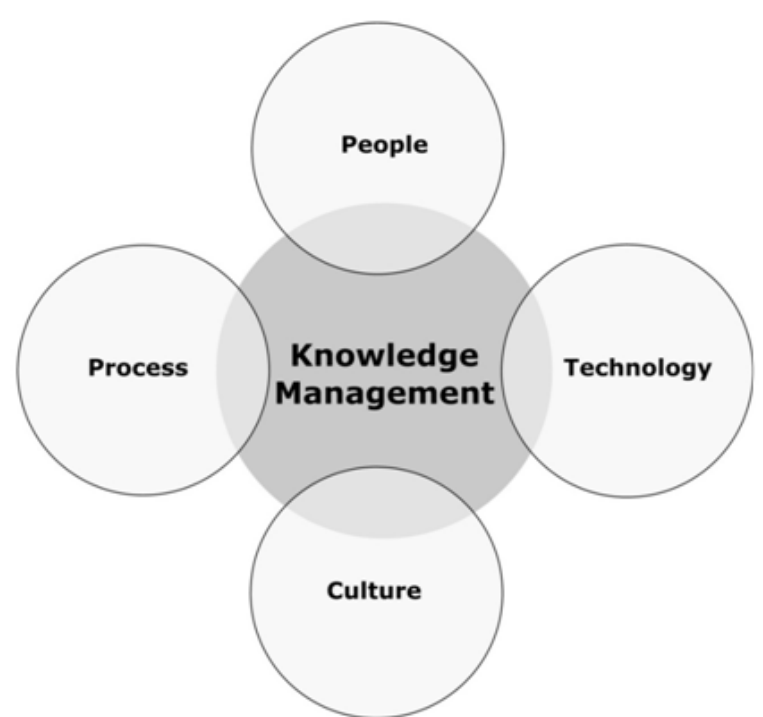

Fig. 1.The four pillars of knowledge management (Modified from original by [4]). 


\section{Human Performance for Service Systems}

The human performance improvement approach in service systems recognizes that any development effort implemented in isolation will produce only limited results. Implementing new service functions or procedure without appropriate training or coaching will not produce improvement. Similarly, any training program implemented without tools and manager support will also have limited impact. According to Wilson Learning [22], Human Performance Improvement (HPI) is a process and creative approach to training that acknowledges the critical role of tools, measurement, and management support which will enhance and extend the impact of learning. There are three elements to service systems training and development effort that are critical to creating maximum results:

1. Establishing a Business Case: Establishing a business case for sustainable performance improvement by linking specific strategic drivers to skill requirements

2. Understanding the Challenge: Making effective decisions about what skills to focus on, selecting delivery methods, and determining how to integrate services into the organization and how to align all key stakeholders to support this approach to performance improvement
3. Creating Integrated Solutions: Creating the performance improvement elements necessary to address the challenge and accomplish the strategy, this includes;

- Developing learning components to deliver the knowledge, skills, and abilities

- Developing work tools and process to support the use of the learning

- Providing organizations with the ability to track the impact of the learning on performance (e.g. business performances analytics)

- Ensuring services planners and managers support and that they are prepared to coach the application of the skills

Quality skills development that makes a measurable impact in the organization requires proper planning and preparation. This involves a structured process of analysis, design, development and implementation. Implementation must be accompanied by the evaluation of the quality and relevance of all learning programs. Figure 2 below shows where the skills training needs analysis fits into the overall process of ensuring that service systems providers have the skills required to fulfill its core processes. This is one of the most important parts of the skills development process. A proper needs analysis will ensure the relevance of the training that is delivered $[9]$.

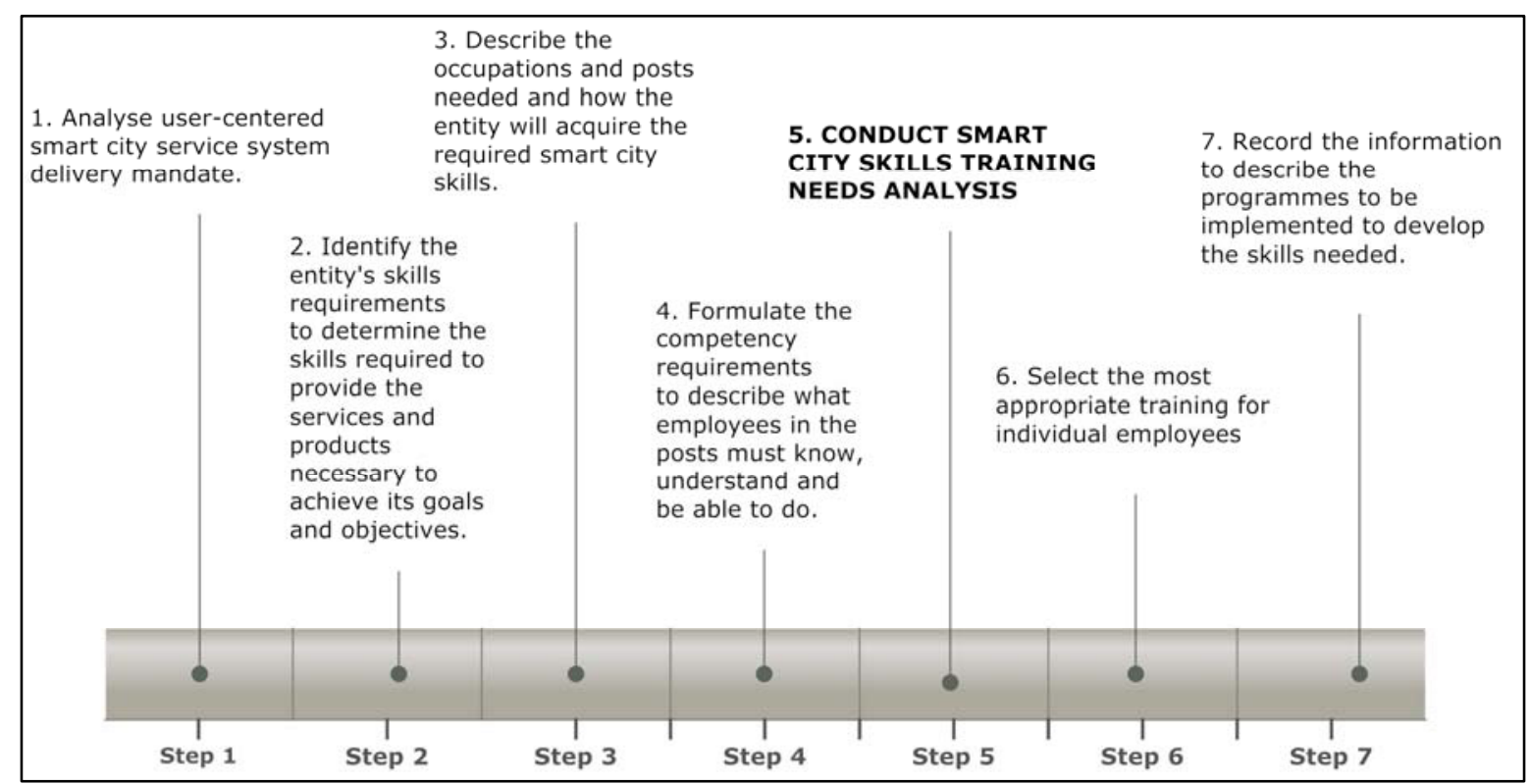

Fig. 2. Sustainable Service systems skills and training needs analysis in service development process [2] 
The purpose of the training needs analysis is to identify the skill gaps and training needs of employees. This information will then be used to determine which employees should be trained in which areas. To determine an employee's training needs planners start with a description of the competence employees should have. They need to know what employees in particular posts should know and understand to be able to perform competently in their jobs (see Figure 2). This will be used as a basis for determining what their current competence is. Once this information is collected, management will be able to determine the training needed to fill the gap between what their competence should be and their current competence level.

Development of a conceptual skills and performances model determines whether the ideas generated about how the systems should look and behave will be perceived clearly by the end user in the manner intended. The framework provided by $[15,16]$ (see Figure 3) illustrates the relationship between the design of a conceptual model and the end user understanding of product usage. In Figure 3, there are three interacting components: the designer, the end-user, and the system. Preece et al. [17] indicated that behind each of these are three interlinking conceptual models:

- The design model - designer's impression of how system should work

- The system component - how the system actually works

- The user's model -the end- user's understanding of how the system works

Preece et al. [17] also indicated that in an ideal world all three conceptual models should map onto each other. End-users should be able to carry out their tasks in the way intended by the designer through interaction with the system. However, if the system image does not make the design model clear to the end-users it is likely that they will end up with an incorrect understanding of system operation and as a result make errors. A sequence of learning about the technology, science, engineering and math (STEM) concepts as well as the processes of research, design, manufacture, construction, testing, and communication, needs to be created. Novice and expert system architects and designers need the background knowledge and skills before they are able to success- fully contribute to a project or design task. The fundamental principle of a competitive services design strategy is to give novice designers a design challenge and let them develop conceptual design solutions and critical thinking skills by applying collaborative design and problem solving [13]. If the design challenge results in something tangible, for example, a manufactured or constructed object, this dramatically increases the motivation of teammates to push themselves. The challenge could take the form of a competition which is an exhilarating experience for novice designers and researchers.

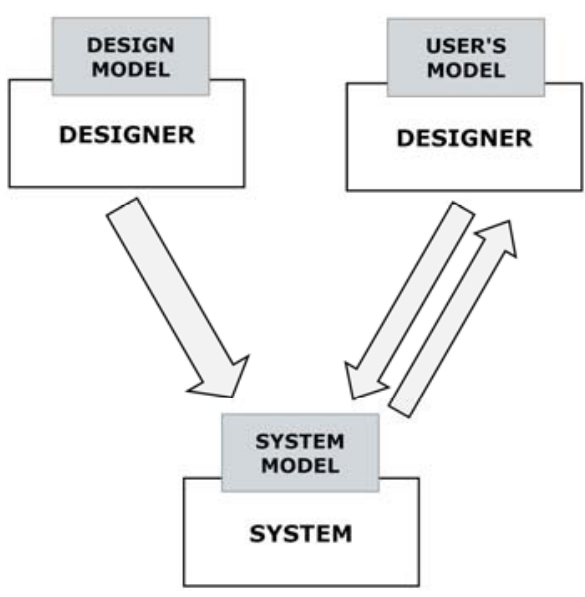

Fig. 3. Conceptual Model Development [17]

\section{The User-centered Sustainable Service System Development Cycle}

The ISO 13407 human-centered design framework is considered the cornerstone for incorporating different business process design techniques, all of which can be merged to support a UCD process. According to the ISO 13407 standard [11], appropriate UCD processes are composed of five iterative steps, which will guarantee the fulfilment of all requirements into the business process design as follows:

- Planning service systems design processes

- Service system context of use

- Requirements specification

- Integration of design solutions

-Service systems evaluation and assessment.

- Planning Service Systems Design Processes

User-centered service system planning plays a crucial role in projects by reducing the risks of sys- 
tem failure, thereby preventing additional costs and disruptions in service. Service system planning will guarantee meeting the process requirements and information flow between service users and the project team. Service system planning helps maintain the maximum integration of the business activities as part of the service system strategy. The first step in planning a service system is to discuss the business needs with stakeholders and users to reach an agreement on how user-centered design techniques can contribute to the service system objectives. In addition, the planning process prioritizes business requirements and highlights the potential benefit gained from including business process activities within the service system development process.

\section{Human Factors Engineering Service Systems Ontologies}

The word "ontology" comes from the Greek ontos, for "being," and logos, for "word." It refers to the study of the categories of things that exist in a domain of knowledge [20]. The idea of ontologies emerged as a means for sharing knowledge. Ontologies rely on well-defined and semantically powerful concepts in artificial intelligence, such as logical statements, reasoning, and rule-based systems. Ontologies enable access to a huge network of machineunderstandable and easily machine-processed human knowledge [21]. As an example of the representation of ontologies, consider the concept of an operations manager. Assume that the concepts used to describe essential knowledge about the concept of operations that are manager, operations, some products of his/her work, namely the company operations the manager was responsible for and departments in which he/she has direct responsibility to manage, and employees, who maintain the company performance. Also, assume that the variety of relationships among these concepts that can be considered may be reduced to just a few of the most essential ones, such as the facts that each operations manager manages some departments, that when giving business decisions he/she check each department performance, and that he also monitors overall business performance. We deliberately avoid in this simple example the numerous kind-of and part-of relationships to other concepts associated with operations management and their detailed responsibilities. These natural-language statements represent the conceptualization of the operations management ontology [1].
The literature describes explorations of potential of software engineering tools and methodologies for ontology development. Pioneering research proposed the unified modeling language (UML), a well-known software modeling language, to be used for ontology development [5]. Gaševic et al. [8], explored further the similarities, differences, and equivalences between UML and ontology languages, as well as the potential of the most recent software engineering initiative called the Model Driven Architecture (MDA)

There are a number of knowledge management representational languages. Web Ontology Language or OWL was adopted by the World Wide Web Consortium (W3C) (http://www.w3c.org); OWL is currently the most popular ontology representation language. OWL facilitates greater machine interpretability of Web content than that supported by XML, RDF, and RDF Schema (RDF-S) by providing additional vocabulary along with a formal semantics [19]. An important feature of the OWL vocabulary is its extreme richness for describing relationships among classes, properties, and individuals. The OWL Web Ontology Language is designed for use by applications that need to process the content of information or "Knowledge" instead of just presenting information to humans. OWL has three increasingly expressive sublanguages: OWL Lite, OWL DL, and OWL Full. Ontologies differ from taxonomies, an ontology is a full specification of a domain. According to [8], a taxonomy is a hierarchical classification of entities within a domain or field of study whereas an ontology is reflection of knowledge and relations between entities within a domain of knowledge and between different domains. Ontologies do not merely serve as a tool for knowledge sharing and knowledge reuse. Ontologies support the concepts and relationships that can exist in a domain and that can be shared and reused. Ontologies reflect the most important part contained which is knowledge and logical reasoning underpinning such hierarchies. However, every ontology also details a taxonomy in a machine readable format. Ontologies facilitate knowledge sharing and reuse across various domains of knowledge. Fikes [7] listed the importance of ontologies in four key application areas: collaboration, interoperation, education, and modeling:

- Collaboration: Ontologies provide a unified knowledge architecture that can be used as a common, shared reference for further development and participation. Researchers and scientists from different domains and backgrounds can talk 
more easily to each other when they have such a stable, consensual knowledge base to rely on.

- Interoperation: Ontologies allow integration of databases from different sources. Distributed applications may need to access different knowledge sources in different formats and in different levels of detail in order to obtain relevant information. Recognizing the same ontology, data conversion and information integration will be easier to allocate.

- Education: Domain experts can use ontologies for education and referencing to share their understanding of the conceptualization and structure of the domain.

- Modeling: Ontologies support the development of knowledge-based applications by providing important reusable building blocks.
Protégé's plug-in-based extensible architecture allows integration with a number of other tools, applications, knowledge bases, and storage formats (e.g. OMG SysML, UML, Visual OWL, XML and XMI for storing knowledge bases) and facilitates concept (class) definition, taxonomies, properties, and restrictions, as well as class instances or actual data in the knowledge base. Furthermore, Protégé has a standard ontology editor GUI with a tab menu for knowledge acquisition and collecting knowledge into a knowledge base conforming to the ontology. The knowledge base can then be used with a problem-solving method to perform various inference or decision making tasks and logical statements and tasks.

Human Factors knowledge management classifications involve two major categories, physical and cognitive aspects of interaction. The cognitive attributes of human factors in knowledge management concentrate on conscious and subconscious mental activities involved in business process decision making [3].

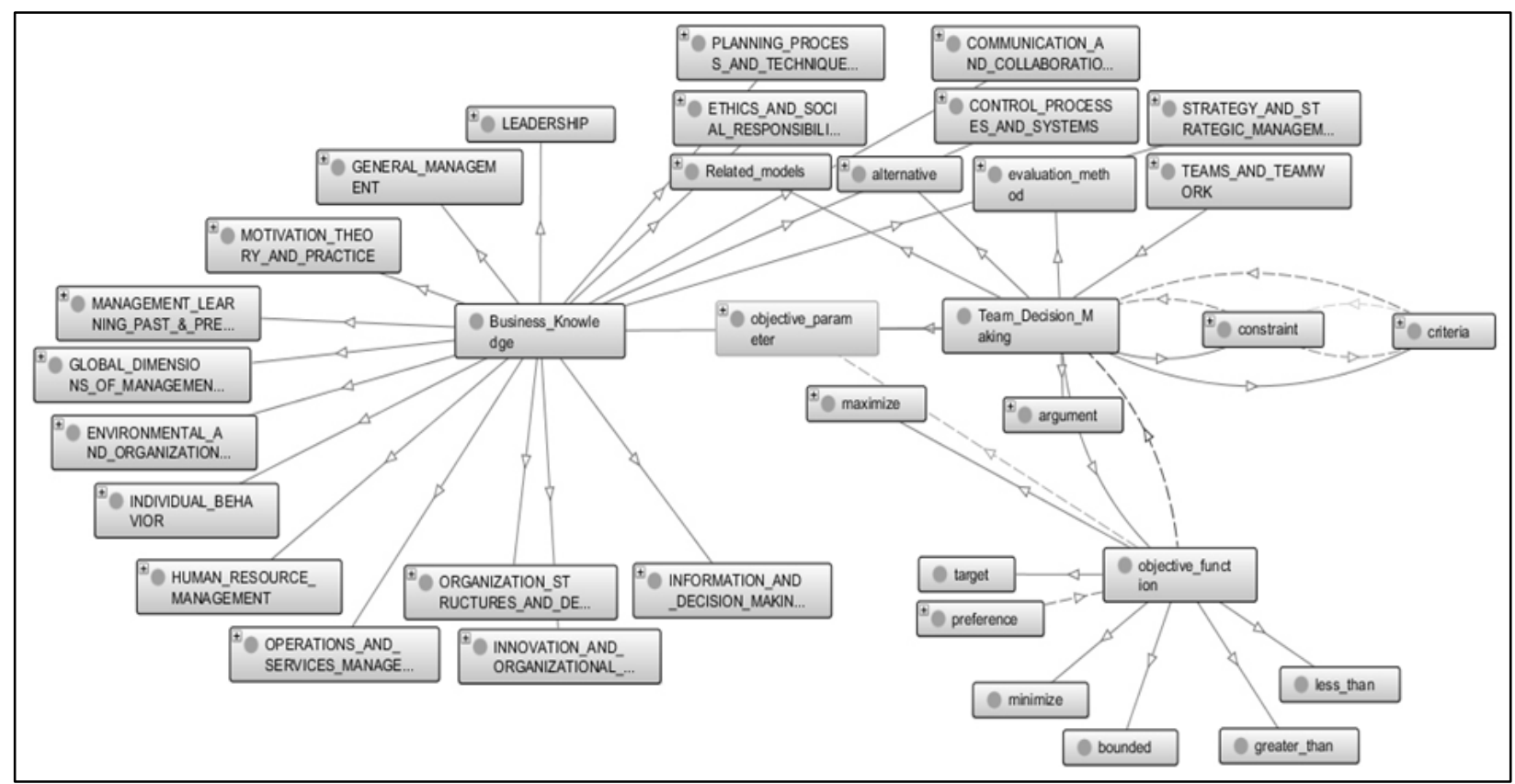

Fig. 4. Model visualization of the business operations management decisions OWL ontology

The standard set of tools for ontology development includes graphical development environments and representation languages. The World Wide Web Consortium (W3C) supports ontology representation on the Semantic Web, also called "Semantic Web languages." Protégé (http://protege.stanford.edu/) is the leading ontology development editor and ODE. Protégé was developed at Stanford University [18].
The knowledge gained in both aspects of cognitive processes is used to create systems and work environments, which help to make people more productive and more satisfied with their work life. A number of different taxonomies exist that break human factors into multiple categories of research. 
A comprehensive human factors and business process modeling ontology framework facilitates the design of agile business process services and systems by capturing the core business knowledge into reusable modules and components [12]. In Figure 4, human factors ontology is connected to business maps and strategy ontologies to aid in decision making and process improvement. This process is supported by the understanding of business roles through a business functions ontology that establishes process frameworks and architecture.

\section{Conclusions}

As a preliminary contribution to the application of knowledge management user-centered design principles and systems engineering process for the design and development of service systems, this paper provides a motivation and quest for an integrated usercentered design approach to systems engineering principles and development lifecycle. While a large number of disciplines and research fields must be integrated towards the development of intelligent and smarter services, considerable advancements achieved in these fields in recent years indicate that the adaptation of these results can lead to highly intelligent and sophisticated yet widely useable systems and services. It is believed that systems engineering (SE) and user-centered design (UCD) approach to design and development should prove useful in supporting and facilitating the development and applications of smarter services in the near future.

\section{References}

[1] T. Z. Ahram, W. Karwowski (2011) "Developing Human Social, Cultural, Behaviour (HSCB) Ontologies: Visualizing \& Modeling Complex Human Interactions", Presented at the Office of Secretary of Defense Human Social, Culture Behavior Modeling (HSCB Focus 2011) (February 8-10, 2011). Chantilly, VA

[2] T. Z. Ahram, W. Karwowski, C. Andrzejczak (2010) "Interactive Management of Human Factors Knowledge for Human Systems Integration", Patricia Ordonez De Pablos (Editor), Electronic Globalized Business and Sustainable Development through IT Management: Strategies and Perspective, IGI Global (ISBN-13: 978-1615206230).

[3] J. Carey (1988). "Human Factors in Management Information Systems", Greenwich, CT: Ablex Publishing Corporation, (pp.7-24).

[4] A . Bourdreau , G. Couillard (2006), Systems Integration and Knowledge Management, Information Systems Management. Vol. 16, Iss. 4.
[5] S. Cranefield (2001), "Networked knowledge representation and exchange using UML and RDF," Journal of Digital Information, Vol. 1, no. 8, article no. 44, 2001-02-15.

[6] K.-P. Fähnrich, and T. Meiren (2007) Service engineering: State of the art and future trends. In: D. Spath and K.-P. Fähnrich, eds. Advances in Service Innovations. Springer, Berlin, pp. 3-16.

[7] R. Fikes, (1998), Multi-use Ontologies, Stanford University [Online]. Available: http://www.ksl.stanford.edu/people/fikes/cs222/1998/Ontolo gies/sld001.htm [Accessed: 2010-08-07]

[8] D. Gaševic, D. Djuric, V. Devedžic, (2006), Model Driven Architecture and Ontology Development, Springer, Berlin.

[9] S. Hattingh (2009). "Skills Development for Performance Improvement: Optimum utilization of the WSP to enhance performance \& service delivery", retrieved from: http://www.learningroadmap.co.za/

[10] J. Hauknes, (1996) Innovation in the Service Economy, STEP group Storgt. 1 N-0155 Oslo, ISSN 0804-8185.

[11] ISO 13407: Human-centered Design Processes for Interactive Systems. International Standards Organization, Geneva, 1999 Also available from the British Standards Institute, London.

[12] W. Janusch (2007). Business Process Discoverer: Semantics Utilised for Process management within and between Enterprises (SUPER). Website: http://www.ip-super.org

[13] W. Karwowski, G. Salvendy, T. Z. Ahram, (2009) "Customercentered Design of Service Organizations”. In: G. Salvendy, W. Karwowski (eds). Introduction to Service Engineering, Paper 9. John Wiley \& Sons, NJ (ISBN-10: 0470382414). (pp.179-206)

[14] J. Nadel, G. Piazza, (2005). Managing the Knowledge Behind Business Decisions through User-Centered Design, Center for Business Knowledge.

[15] D. Norman, (1988). The Design of Everyday Things. New York: Basic Books.

[16] D. Norman, (1993). Things That Make Us Smart. Reading, MA: Addison-Wesley.

[17] J. Preece, Y. Rogers, H. Sharp, , (2002). Interaction Design: beyond human-computer interaction, New York, NY: J - Wiley \& Sons

[18] Protégé Ontologies Library (2010), ProtegeOntologiesLibrary [Online]. Available: http://protegewiki.stanford.edu/wiki/Protege_Ontology_Libra ry [Accessed: 2010, August 8].

[19] M. K. Smith, C. Welty, and D. McGuinness (2004). OWL Web Ontology Language Guide, Editors, W3C Recommendation, http://www.w3.org/TR/2004/REC-owl-guide$20040210 /$. Latest version available at http://www.w3.org/TR/owl-guide/ .

[20] J.F. Sowa, (2000), Knowledge Representation: Logical, Philosophical, and Computational Foundations, Brooks Cole, Pacific Grove, CA.

[21] R. Gruber (1993). A Translation Approach to Portable Ontology Specifications. Knowledge Acquisition, 5(2), 1993, pp. 199-220.

[22] Wilson Learning Worldwide Website: http://wilsonlearning.com/ 